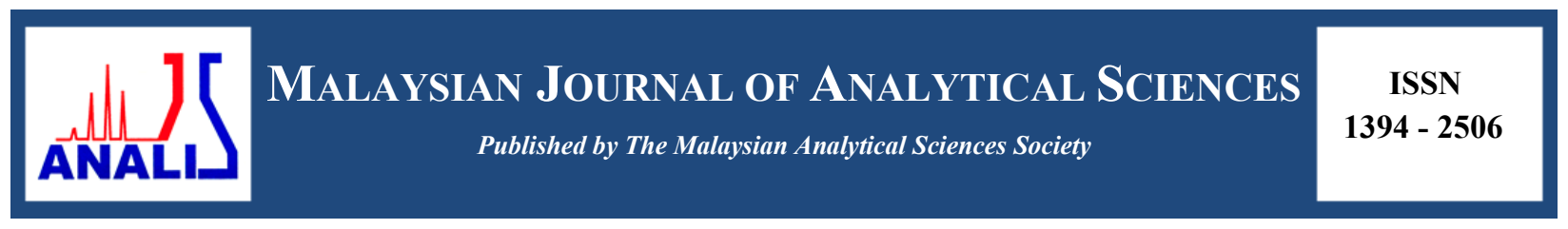

\title{
THE CHEMICAL CONSTITUENTS FROM YOUNG TUBERS of Hydnophytum formicarum
}

\author{
(Juzuk Kimia Daripada Tuber Muda Hydnophytum formicarum) \\ Nur Shafiqa Abdullah, Wan Yaacob Wan Ahmad*, Noor Aziiraa Sabri \\ School of Chemical Sciences and Food Technology, \\ Faculty of Science and Technology \\ Universiti Kebangsaan Malaysia, 43600 UKM Bangi, Selangor, Malaysia \\ *Corresponding author: wanyaa@ukm.edu.my
}

Received: 6 December 2016; Accepted: 16 January 2017

\begin{abstract}
The fine powders of young tubers of Hydnophytum formicarum was extracted by Soxhlet methanol extraction for 18 hours in three days continuously. The filtrate then was evaporated by rotary evaporator until it became concentrated solution. The fractionation and purification of the extract by vacuum liquid chromatography and radial chromatography has led to the discovery of four compounds namely sinapinic acid, $\beta$-sitosterol acetate, $\beta$-sitosterol and stigmasterol. Structures of the compounds were established by interpreting mass spectral data, ${ }^{1} \mathrm{H}$ and ${ }^{13} \mathrm{C}-\mathrm{APT} \mathrm{NMR}$, Infrared and by comparison with literature data.
\end{abstract}

Keywords: Hydnophytum formicarum, sinapinic acid, $\beta$-sitosterol acetate, $\beta$-sitosterol, stigmasterol

Abstrak

Serbuk halus tuber muda Hydnophytum formicarum telah diekstrak melalui pengekstrakan Soxhlet selama 18 jam dalam tiga hari secara berturutan. Hasil penurasan kemudian telah disejat menggunakan penyejat berputar sehingga menjadi larutan yang likat. Pemfraksian dan penulenan ekstrak menggunakan kromatografi cecair vakum dan kromatografi radial membawa kepada penemuan empat sebatian yang bernama asid sinapinik, $\beta$-sitosterol asetat, $\beta$-sitosterol dan stigmasterol. Struktur sebatian ini telah ditentukan dengan mentafsirkan data spektrum jisim, RMN ${ }^{1} \mathrm{H}$ dan ${ }^{13} \mathrm{C}$-APT, Inframerah serta secara membandingkannya dengan data kepustakaan.

Kata kunci: Hydnophytum formicarum, asid sinapinik, $\beta$-sitosterol asetat, $\beta$-sitosterol, stigmasterol

\section{Introduction}

Rubiaceae is the largest plant family containing 10,700 species and 637 genera that consists mainly of tropical woody plants with great pharmacological potential [1]. The previous phytochemical study of all Rubiaceae species, published from 1990 to 2014, stated that the most common secondary metabolites of this family were iridoids, anthraquinones, triterpenes and indole alkaloids as well as other varying alkaloid subclasses [2]. One of the species, Hydnophytum formicarum or ant plant, is a tuberous ephyphytic of medicinal plant known in Malaysia as Raja Satong or Kepala Beruk or Sarang Semut. It is commonly found in Southeast Asia (Malaysia and Indonesia), Papua New Guinea and Pacific Islands [3].

The objectives of this study are to isolate compounds from the young tubers of Hydnophytum formicarum by extraction and fractionation using several chromatographic techniques as well as to elucidate structures of pure 
compounds. Traditionally, the decoction of Hydnophytum formicarum tubers was used as a drink for liver and intestinal complaints. Soxhlet extraction with methanol was employed in this study in view of the use of the tubers by local people for medicinal purposes by boiling in water. Moreover, more constituents can be extracted by Soxhlet compared to maceration. Darwis et al. reported flavonoid, phenolic, aldehydes, ketones, terpenoids and tannin in the ethanol Hydnophytum formicarum extract [4]. Previous studies reported several compounds from Hydnophytum formicarum such as $\beta$-sitosterol [5], 7,3',5'-trihydroxyflavanone [6], stigmasterol, protocatechualdehyde, butin, butein, and isoliquiritigenin [3]. Figure 1 below shows a young tuber of Hydnophytum formicarum. The tubers of Hydnophytum formicarum have been scientifically proven to contain bioactive constituents such as antioxidant and antimicrobial [3] and antiproliferative [7].

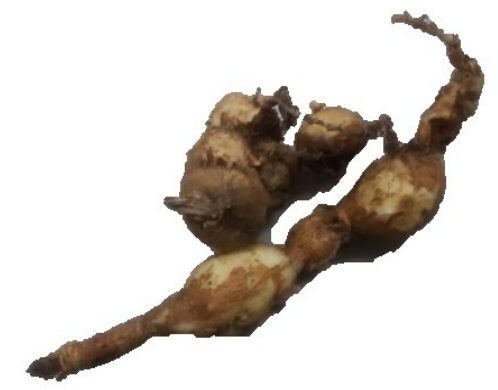

Figure 1. Young tuber of Hydnophytum formicarum

\section{General}

\section{Materials and Methods}

The ${ }^{1} \mathrm{H}$ and ${ }^{13} \mathrm{C}$-APT NMR spectra were recorded with Avance III $700 \mathrm{MHz}$ Bruker in $\mathrm{CDCl}_{3}$ with the chemical shifts, $\delta$, in ppm and the values of coupling constants, $J$, in Hz. LC-MSToF spectra were taken on the Dionex/Bruker Micro ToFQ and FTIR spectra were recorded on the Perkin-Elmer Spectrum 400 FT-IR/FT-NIR.

\section{Plant material}

Young tubers of Hydnophytum formicarum were bought in April 2015 from a seller at Kampung Lak Lok, Jertih, Besut, Terengganu. A voucher specimen (UKMB 40312) was deposited at the Herbarium of Universiti Kebangsaan Malaysia Bangi (UKMB).

\section{Extraction and isolation}

The air-dried young tubers of Hydnophytum formicarum were cut into small pieces and ground into fine powder by using grinding machine. The powder $(1.04 \mathrm{~kg})$ was then extracted with methanol by using Soxhlet for 6 hours in three days to give $15.0 \mathrm{~g}(1.44 \%)$ of dark-brown extract after solvent removal by rotary evaporator. The extract was subjected to vacuum liquid chromatography (VLC) by using silica gel 7747 (Merck) eluted with increasing polarity of $n$-hexane and ethyl acetate. The eluents were combined based on their silica gel thin layer chromatography (TLC) (Merck 5554) profile to give seven fractions (A-G). Fraction A and B was washed with $n$-hexane to give stigmasterol $(37.0 \mathrm{mg})$ and $\beta$-sitosterol $(22.0 \mathrm{mg})$. Fraction $\mathrm{E}(0.84 \mathrm{~g})$ was further purified by column chromatography (CC) using $n$-hexane-ethyl acetate (7:3) to yield six fractions (E1-E6). The E6 was pure to give sinapinic acid $(29.0 \mathrm{mg})$. Fraction C (1.2 g) was further purified by radial chromatography (RC) using $n$-hexaneethyl acetate (7:3) to produce $\beta$-sitosterol acetate $(23.7 \mathrm{mg})$.

\section{Characterization study}

\section{Results and Discussion}

Sinapinic acid $(29.0 \mathrm{mg})$ : white powder; $\mathrm{Rf}=0.70$ ( $n$-hexane-ethyl acetate, 7:3); ESI-MS (m/z): $247.2077[\mathrm{M}+\mathrm{Na}]^{+}$, $\mathrm{C}_{11} \mathrm{H}_{12} \mathrm{O}_{5}$; FTIR (ATR) $\mathrm{cm}^{-1}: 3412(\mathrm{O}-\mathrm{H}), 2916$ and $2849\left(\mathrm{Csp} p^{3}-\mathrm{H}\right), 1711(\mathrm{C}=\mathrm{O}$ carboxylic acid), 1268 and 1158 (C-O), 1463-1632 (C-C aromatic), 718-1032 (Csp $\left.{ }^{2}-\mathrm{H}\right)$. 
$\beta$-Sitosterol acetate $(23.7 \mathrm{mg})$ : white needles; $\mathrm{Rf}=0.81$ ( $n$-hexane-ethyl acetate, 7:3); ESI-MS ( $\mathrm{m} / \mathrm{z}): 479.3404$ $[\mathrm{M}+\mathrm{Na}]^{+}, \mathrm{C}_{31} \mathrm{H}_{52} \mathrm{O}_{2}$; FTIR (ATR) $\mathrm{cm}^{-1}: 2928$ and $2868\left(\mathrm{Csp}^{3}-\mathrm{H}\right), 1674(\mathrm{C}=\mathrm{O}), 1458,1378\left(\mathrm{Csp}^{3}-\mathrm{H}\right)$.

$\beta$-Sitosterol (22.0 mg): white needles; $\mathrm{Rf}=0.94$ ( $n$-hexane-ethyl acetate, 8:2); ESI-MS (m/z): $437.6012[\mathrm{M}+\mathrm{Na}]^{+}$, $\mathrm{C}_{29} \mathrm{H}_{50} \mathrm{O}$; FTIR (ATR) $\mathrm{cm}^{-1}: 3374(\mathrm{O}-\mathrm{H}), 2933$ and $2866\left(\mathrm{Csp}^{3}-\mathrm{H}\right), 1052(\mathrm{C}-\mathrm{O}), 801(\mathrm{O}-\mathrm{H}), 1463$ and $1378\left(\mathrm{Csp}^{3}\right.$ $\mathrm{H}), 801-957\left(\mathrm{Csp} p^{2}-\mathrm{H}\right)$.

Stigmasterol (37.0 mg): white needles; $\mathrm{Rf}=0.90$ ( $n$-hexane-ethyl acetate, 9:1); ESI-MS (m/z): 435.4306 [M+Na] $]^{+}$, $\mathrm{C}_{29} \mathrm{H}_{48} \mathrm{O}$; FTIR (ATR) cm ${ }^{-1}: 3330(\mathrm{O}-\mathrm{H}), 2931$ and $2869\left(\mathrm{Csp}^{3}-\mathrm{H}\right), 1052(\mathrm{C}-\mathrm{O}), 800(\mathrm{O}-\mathrm{H}), 1644(\mathrm{C}=\mathrm{C}), 1463$, $1376\left(\mathrm{Csp}^{3}-\mathrm{H}\right)$.<smiles>COc1cc(/C=C/C(=O)O)cc(OC)c1O</smiles>

(1)

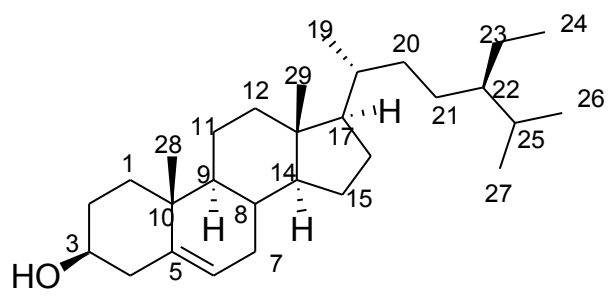

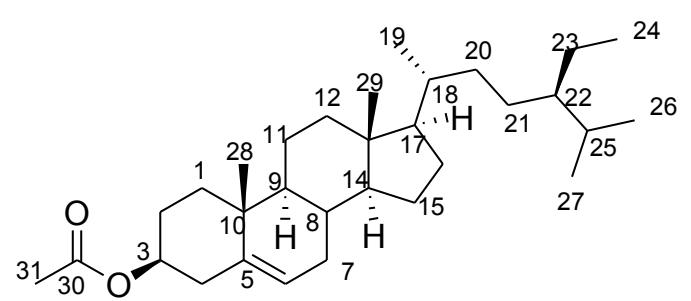

(2)

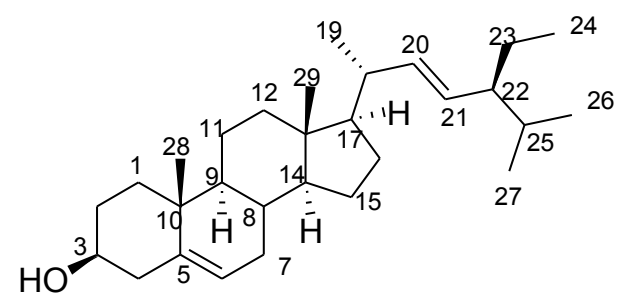

(4)

Figure 2. Molecular structures of sinapinic acid (1), $\beta$-sitosterol acetate (2), $\beta$-sitosterol (3) and stigmasterol (4)

Table 1. ${ }^{1} \mathrm{H}$ and ${ }^{13} \mathrm{C}$-APT NMR $\left(700 \mathrm{MHz}, \mathrm{CDCl}_{3}\right)$ spectral data of sinapinic acid and literature

\begin{tabular}{lcccl}
\hline \multirow{2}{*}{ No. } & \multicolumn{3}{c}{ Sinapinic acid } & \multicolumn{2}{c}{ Sinapinic acid* } \\
\cline { 2 - 5 } & $\boldsymbol{\delta}_{\mathbf{C}}$ & $\boldsymbol{\delta}_{\mathbf{H}}(\mathbf{\Sigma H}, \boldsymbol{m}, \boldsymbol{J})$ & $\boldsymbol{\delta}_{\mathbf{C}}$ & $\boldsymbol{\delta}_{\mathbf{H}}(\mathbf{\Sigma H}, \boldsymbol{m}, \boldsymbol{J})$ \\
\hline 1 & 139.9 & & 139.2 & \\
2 & 106.0 & $7.04(2 \mathrm{H}, s)$ & 106.6 & $7.01(2 \mathrm{H}, s)$ \\
3 & 148.3 & & 148.4 & \\
4 & 145.6 & & 146.0 & \\
$1^{\prime}$ & 115.6 & $6.29(1 \mathrm{H}, d, J=15.9 \mathrm{~Hz})$ & 116.1 & $6.39(1 \mathrm{H}, d, J=15.8 \mathrm{~Hz})$ \\
$2^{\prime}$ & 127.0 & $7.61(1 \mathrm{H}, d, J=15.9 \mathrm{~Hz})$ & 126.0 & $7.58(1 \mathrm{H}, d, J=15.9 \mathrm{~Hz})$ \\
$3^{\prime}$ & 167.4 & & 167.9 & \\
$4^{\prime}$ & 56.0 & $3.93(6 \mathrm{H}, s)$ & 56.5 & $3.91(6 \mathrm{H}, s)$ \\
\hline
\end{tabular}

Source: *[8] 
Comparison of ${ }^{1} \mathrm{H}$ and ${ }^{13} \mathrm{C}$-APT NMR spectral data of sinapinic acid (Table 1) with those literature data confirmed that both are of the same compound [8]. The ESI-MS gave molecular ion at $\mathrm{m} / \mathrm{z} 247.2077[\mathrm{M}+\mathrm{Na}]^{+}$, which corresponded to the molecular formula $\mathrm{C}_{11} \mathrm{H}_{12} \mathrm{O}_{5}$. Sinapinic acid and its ester had been isolated from Rubiaceae family such as Gardenia jasminoides [12] and Mussaenda incana [13] supports its existence in Hydnophytum formicarum.

Similarity of ${ }^{1} \mathrm{H}$ and ${ }^{13} \mathrm{C}$-APT NMR spectral data of $\beta$-sitosterol acetate (Table 2) with those of Gohari et al. and Ran et al. confirmed that they are of the same structure. The ESI-MS showed molecular ion at $\mathrm{m} / \mathrm{z} 479.3404$ $[\mathrm{M}+\mathrm{Na}]^{+}$, which is in accordance with the molecular formula $\mathrm{C}_{31} \mathrm{H}_{50} \mathrm{O}_{2}$. To the best of our knowledge, this is the first time $\beta$-sitosterol acetate has been isolated from Hydnophytum formicarum, and this sterol has never been reported before from any other genus of Rubiaceae.

The NMR spectral data $\left({ }^{1} \mathrm{H}\right.$ and ${ }^{13} \mathrm{C}$-APT) of $\beta$-sitosterol and stigmasterol (Table 3$)$ in agreement with those of literature data [11]. The ESI-MS exhibited respective molecular ions at $\mathrm{m} / \mathrm{z} 437.6012[\mathrm{M}+\mathrm{Na}]^{+}$and 435.4306 $[\mathrm{M}+\mathrm{Na}]^{+}$, which matched the molecular formulas of $\mathrm{C}_{29} \mathrm{H}_{50} \mathrm{O}$ ( $\beta$-sitosterol) and $\mathrm{C}_{29} \mathrm{H}_{48} \mathrm{O}$ (stigmasterol). Besides frequent identification of these sterols as common compounds in many plants species, they are also found in the Rubiaceae family such as Fadogia homblei [14] and Rudgea jasminoides [15].

Table 2. ${ }^{1} \mathrm{H}$ and ${ }^{13} \mathrm{C}$-APT NMR $\left(700 \mathrm{MHz}, \mathrm{CDCl}_{3}\right)$ spectral data of $\beta$-sitosterol acetate and literature

\begin{tabular}{|c|c|c|c|c|}
\hline \multirow[t]{2}{*}{ No. } & \multicolumn{2}{|r|}{$\beta$-Sitosterol acetate } & \multicolumn{2}{|r|}{$\beta$-Sitosterol acetate } \\
\hline & $\boldsymbol{\delta}_{\mathrm{C}}$ & $\delta_{\mathrm{H}}(\Sigma \mathrm{H}, m, J \mathbf{H z})$ & $\boldsymbol{\delta}_{\mathrm{C}}{ }^{*}$ & $\delta_{\mathrm{H}}(\Sigma \mathrm{H}, m, J \mathrm{~Hz})^{* * *}$ \\
\hline 1 & 36.3 & & 37.0 & \\
\hline 2 & 27.5 & & 27.8 & \\
\hline 3 & 72.0 & $4.67(1 \mathrm{H}, m)$ & 73.9 & $4.55(1 \mathrm{H}, m)$ \\
\hline 4 & 38.9 & & 38.1 & \\
\hline 5 & 138.4 & & 138.2 & \\
\hline 6 & 123.9 & $5.15(1 \mathrm{H}, d, J=4.5)$ & 122.5 & $5.37(1 \mathrm{H}, d, J=4.2)$ \\
\hline 7 & 32.2 & & 32.0 & \\
\hline 8 & 31.5 & & 31.9 & \\
\hline 9 & 50.3 & & 50.1 & \\
\hline 10 & 36.3 & & 36.6 & \\
\hline 11 & 21.2 & & 21.0 & \\
\hline 12 & 39.8 & & 39.6 & \\
\hline 13 & 42.6 & & 42.2 & \\
\hline 14 & 56.2 & & 56.8 & \\
\hline 15 & 24.4 & & 24.4 & \\
\hline 16 & 28.4 & & 28.8 & \\
\hline 17 & 56.1 & & 56.0 & \\
\hline 18 & 40.4 & & 40.4 & \\
\hline 19 & 21.5 & $0.91(3 \mathrm{H}, d, J=6.5)$ & 21.5 & $0.91(3 \mathrm{H}, d, J=5.0)$ \\
\hline 20 & 34.2 & & 34.0 & \\
\hline 21 & 26.2 & & 26.1 & \\
\hline 22 & 51.2 & & 51.2 & \\
\hline 23 & 25.4 & & 25.4 & \\
\hline
\end{tabular}


Table 2 (cont'd). ${ }^{1} \mathrm{H}$ and ${ }^{13} \mathrm{C}$-APT NMR $\left(700 \mathrm{MHz}, \mathrm{CDCl}_{3}\right.$ ) spectral data of $\beta$-sitosterol acetate and literature

\begin{tabular}{lcccl}
\hline \multirow{2}{*}{ No. } & \multicolumn{2}{c}{$\boldsymbol{\beta}$-Sitosterol acetate } & \multicolumn{2}{c}{$\boldsymbol{\beta}$-Sitosterol acetate } \\
\cline { 2 - 5 } & $\boldsymbol{\delta}_{\mathbf{C}}$ & $\boldsymbol{\delta}_{\mathbf{H}}(\boldsymbol{\Sigma} \mathbf{H}, \boldsymbol{m}, \boldsymbol{J} \mathbf{H z})$ & $\boldsymbol{\delta}_{\mathbf{C}} *$ & $\boldsymbol{\delta}_{\mathbf{H}}(\boldsymbol{\Sigma} \mathbf{H}, \boldsymbol{m}, \boldsymbol{J} \mathbf{H z})^{* *}$ \\
\hline 24 & 12.2 & & 12.2 & \\
25 & 30.0 & & 31.8 & \\
26 & 19.2 & $0.85(3 \mathrm{H}, d, J=7.0)$ & 19.0 & $0.83(3 \mathrm{H}, d, J=6.7)$ \\
27 & 21.2 & & 21.2 & \\
28 & 19.3 & & 19.3 & \\
29 & 12.1 & $1.83(3 \mathrm{H}, s)$ & 12.0 & $1.00(3 \mathrm{H}, s)$ \\
30 & 172.0 & & 170.2 & \\
31 & 21.2 & $2.03(3 \mathrm{H}, s)$ & 21.4 & $2.03(3 \mathrm{H}, s)$ \\
\hline
\end{tabular}

Source: $*[9]$ and $* *[10]$

Table 3. ${ }^{1} \mathrm{H}$ and ${ }^{13} \mathrm{C}$-APT NMR (700 MHz, $\left.\mathrm{CDCl}_{3}\right)$ spectral data of stigmasterol and $\beta$-sitosterol and literature

\begin{tabular}{|c|c|c|c|c|c|c|c|c|}
\hline \multirow{2}{*}{ No. } & \multicolumn{2}{|c|}{ Stigmasterol } & \multicolumn{2}{|c|}{ Stigmasterol* } & \multicolumn{2}{|c|}{$\beta$-Sitosterol } & \multicolumn{2}{|c|}{$\beta$-Sitosterol* } \\
\hline & $\boldsymbol{\delta}_{\mathrm{C}}$ & $\begin{array}{l}\delta_{H} \\
H z)\end{array}(\Sigma H, m, J$ & $\boldsymbol{\delta}_{\mathrm{C}}$ & $\begin{array}{l}\delta_{\mathrm{H}}(\Sigma \mathrm{H}, m, J \\
\mathrm{Hz})\end{array}$ & $\boldsymbol{\delta}_{\mathrm{C}}$ & $\begin{array}{l}\delta_{\mathrm{H}}(\Sigma \mathrm{H}, m, J \\
\mathrm{Hz})\end{array}$ & $\boldsymbol{\delta}_{\mathrm{C}}$ & $\begin{array}{l}\delta_{\mathrm{H}}(\Sigma \mathrm{H}, m, J \\
\mathrm{Hz})\end{array}$ \\
\hline 1 & 37.4 & & 37.6 & & 37.3 & & 37.5 & \\
\hline 2 & 32.0 & & 32.1 & & 32.0 & & 31.9 & \\
\hline 3 & 71.9 & $3.52(1 \mathrm{H}, m)$ & 72.1 & $3.52(1 \mathrm{H}, m)$ & 71.9 & $3.52(1 \mathrm{H}, m)$ & 72.0 & $\begin{array}{l}3.53(1 \mathrm{H}, t d d, J \\
=4.5,4.2,3.8)\end{array}$ \\
\hline 4 & 42.4 & & 42.4 & & 42.4 & & 42.5 & \\
\hline 5 & 140.9 & & 141.1 & & 140.8 & & 140.9 & \\
\hline 6 & 121.8 & $\begin{array}{l}5.34(1 \mathrm{H}, t, J= \\
2.6)\end{array}$ & 121.8 & $\begin{array}{l}5.34(1 \mathrm{H}, \mathrm{t}, J \\
=2.1)\end{array}$ & 121.8 & $\begin{array}{l}5.34(1 \mathrm{H}, t, J \\
=2.4)\end{array}$ & 121.9 & $\begin{array}{l}5.36(1 \mathrm{H}, t, J \\
=6.4)\end{array}$ \\
\hline 7 & 32.0 & & 31.8 & & 32.0 & & 32.1 & \\
\hline 8 & 32.0 & & 31.8 & & 32.0 & & 32.1 & \\
\hline 9 & 50.2 & & 50.2 & & 50.2 & & 50.3 & \\
\hline 10 & 36.6 & & 36.6 & & 36.2 & & 36.7 & \\
\hline 11 & 21.2 & & 21.5 & & 21.1 & & 21.3 & \\
\hline 12 & 39.9 & & 39.9 & & 39.8 & & 39.9 & \\
\hline 13 & 42.4 & & 42.4 & & 42.4 & & 42.6 & \\
\hline 14 & 56.9 & & 56.8 & & 56.8 & & 56.9 & \\
\hline 15 & 24.4 & & 24.4 & & 26.1 & & 26.3 & \\
\hline 16 & 29.3 & & 29.3 & & 28.3 & & 28.5 & \\
\hline 17 & 56.2 & & 56.2 & & 56.1 & & 56.3 & \\
\hline 18 & 40.6 & & 40.6 & & 36.2 & & 36.3 & \\
\hline 19 & 21.2 & $\begin{array}{l}0.84(3 \mathrm{H}, d, J= \\
4.1)\end{array}$ & 21.7 & $\begin{array}{l}1.13(3 \mathrm{H}, d, J \\
=6.8)\end{array}$ & 19.1 & $\begin{array}{l}0.91(3 \mathrm{H}, d, J \\
=5.0)\end{array}$ & 19.2 & $\begin{array}{l}0.93(3 \mathrm{H}, d, J \\
=6.5)\end{array}$ \\
\hline 20 & 138.4 & $\begin{array}{l}5.02(1 \mathrm{H}, d d, J= \\
15.2,8.7)\end{array}$ & 138.7 & $5.05(1 \mathrm{H}, m)$ & 34.0 & & 34.2 & \\
\hline
\end{tabular}


Table 3 (cont'd). ${ }^{1} \mathrm{H}$ and ${ }^{13} \mathrm{C}$-APT NMR $\left(700 \mathrm{MHz}, \mathrm{CDCl}_{3}\right)$ spectral data of stigmasterol and $\beta$-sitosterol and literature

\begin{tabular}{|c|c|c|c|c|c|c|c|c|}
\hline \multirow{2}{*}{ No. } & \multicolumn{2}{|c|}{ Stigmasterol } & \multicolumn{2}{|c|}{ Stigmasterol* } & \multicolumn{2}{|c|}{$\beta$-Sitosterol } & \multicolumn{2}{|c|}{$\beta$-Sitosterol* } \\
\hline & $\boldsymbol{\delta}_{\mathrm{C}}$ & $\begin{array}{l}\delta_{H} \\
H z)\end{array}(\Sigma H, m, J$ & $\boldsymbol{\delta}_{\mathrm{C}}$ & $\begin{array}{l}\delta_{\mathrm{H}}(\Sigma \mathrm{H}, m, J \\
\mathrm{Hz})\end{array}$ & $\boldsymbol{\delta}_{\mathrm{C}}$ & $\begin{array}{l}\delta_{\mathrm{H}}(\Sigma \mathrm{H}, m, J \\
\mathrm{Hz})\end{array}$ & $\boldsymbol{\delta}_{\mathrm{C}}$ & $\begin{array}{l}\delta_{H}(\Sigma H, m, J \\
H z)\end{array}$ \\
\hline 21 & 129.4 & $\begin{array}{l}5.15(1 \mathrm{H}, d d, J= \\
15.2,8.7)\end{array}$ & 139.6 & $5.01(1 \mathrm{H}, m)$ & 26.1 & & 26.3 & \\
\hline 22 & 45.9 & & 46.1 & & 45.9 & & 46.1 & \\
\hline 23 & 25.5 & & 25.4 & & 23.1 & & 23.3 & \\
\hline 24 & 12.1 & $\begin{array}{l}0.86(3 \mathrm{H}, t, J= \\
7.1)\end{array}$ & 12.1 & $\begin{array}{l}0.82(3 \mathrm{H}, t, J \\
=6.7)\end{array}$ & 12.0 & $\begin{array}{l}0.84(3 \mathrm{H}, t, J \\
=7.2)\end{array}$ & 12.2 & $\begin{array}{l}0.84(3 \mathrm{H}, t, J \\
=7.2)\end{array}$ \\
\hline 25 & 29.3 & & 29.6 & & 29.2 & & 29.4 & \\
\hline 26 & 19.9 & $\begin{array}{l}0.85(3 \mathrm{H}, d, J= \\
6.7)\end{array}$ & 20.2 & $\begin{array}{l}0.80(3 \mathrm{H}, d, J \\
=6.6)\end{array}$ & 19.8 & $\begin{array}{l}0.83(3 \mathrm{H}, d, J \\
=6.7)\end{array}$ & 20.1 & $\begin{array}{l}0.83(3 \mathrm{H}, d, J \\
=6.4)\end{array}$ \\
\hline 27 & 19.9 & $\begin{array}{l}0.82(3 \mathrm{H}, d, J= \\
6.7)\end{array}$ & 19.8 & $\begin{array}{l}0.89(3 \mathrm{H}, d, J \\
=6.6)\end{array}$ & 19.9 & $\begin{array}{l}0.81(3 \mathrm{H}, d, J \\
=6.7)\end{array}$ & 19.6 & $\begin{array}{l}0.81(3 \mathrm{H}, d, J \\
=6.4)\end{array}$ \\
\hline 28 & 18.8 & $0.69(3 \mathrm{H}, s)$ & 18.9 & $0.89(3 \mathrm{H}, s)$ & 19.1 & $0.67(3 \mathrm{H}, s)$ & 19.0 & $0.68(3 \mathrm{H}, \mathrm{s})$ \\
\hline 29 & 12.0 & $1.01(3 \mathrm{H}, s)$ & 12.2 & $1.22(3 \mathrm{H}, s)$ & 12.0 & $1.00(3 \mathrm{H}, s)$ & 12.0 & $1.01(3 \mathrm{H}, \mathrm{s})$ \\
\hline
\end{tabular}

Source: *[11]

\section{Conclusion}

Chromatographic separation of Soxhlet methanol extract of Hydnophytum formicarum young tubers yielded four compounds namely sinapinic acid, $\beta$-sitosterol acetate, $\beta$-sitosterol and stigmasterol. Sinapinic acid and $\beta$-sitosterol acetate can be used to evaluate their biological activities such as antimicrobial, antioxidant, anti-cancer and antiinflammatory properties.

\section{Acknowledgement}

We would like to thank the Ministry of Education, Malaysia and Univeristi Kebangsaan Malaysia (UKM) for financial support under the DLP-2013-018 grant. Special thanks to School of Chemical Sciences and Food Technology, Faculty of Science and Technology, UKM and Centre for Research and Innovation Management (CRIM) UKM for the technical support on NMR, LC-MSToF and FTIR instrumentation.

\section{References}

1. Ahmad, R., Mahbob, E. N. M., Noor, Z. M., Ismail, N. H., Lajis, N. H. and Shaari K. (2010). Evaluation of antioxidant potential of medicinal plants from Malaysian Rubiaceae (subfamily rubioideae). African Journal of Biotechnology, 9(46): 7948 - 7954.

2. Martins, D. and Nunez, C.V. (2015). Secondary metabolites from Rubiaceae species. Molecules, 20(7): 13422 13495.

3. Prachayasittikul, S., Buraparuangsang, P., Worachartcheewan, A., Isarankura-Na-Ayudhya, C., Ruchirawat, S. and Prachayasittikul, V. (2008). Antimicrobial and antioxidative activities of bioactive constituents from Hydnophytum formicarum Jack. Molecules 13(4): 904 - 921.

4. Darwis, D., Hertiani, T. and Samito, E. (2014). The effects of Hydnophytum formicarum ethanolic extract toward lymphocyte, vero and T47d cells proliferation in vitro. Journal of Applied Pharmaceutical Science, 4(6): $103-109$.

5. Prachayasittikul, S., Pingaew, R., Yamkamon, V., Worachartcheewan, A., Wanwimolruk, S., Ruchirawat, S. and Prachayasittikul, V. (2012). Chemical constituents and antioxidant activity of Hydnophytum formicarum Jack. International Journal of Pharmacology, 8(5): 440 - 444. 
6. Abdullah, H., Pihie, A. H. L., Hohmann, J. and Molnar, J. (2010). A natural compound from Hydnophytum formicarum induces apoptosis of MCF-7 cells via up-regulation of Bax. Cancer Cell International, 10: 1-6.

7. Ueda, J., U., Tezuka, Y., Banskota, A.H., Le Tran, Q., Kim, Q., Harimaya, Y., Saiki, I. and Kadota, S. (2002). Antiproliferative activity of Vietnamese medicinal plants. Biological and Pharmaceutical Bulletin, 25(6): 753 760.

8. Cai, R., Arntfield, S. D. and Charlton, J. L. (1999). Structural changes of sinapinic acid and sinapine bisulfate during autoclaving with respect to the development of colored substances. Journal of the American Oil Chemist's Society 76: 433 - 441.

9. Gohari, A. R., Saeidnia, S., Malmir, M., Yazdanpanah, M. and Ajani, Y. (2011). Sterols and flavonoids of Lomatopodium staurophyllum. Journal of Medicinal Plants, 10(39): 43 - 48.

10. Ran, T., Cheng, Z.W. \& Zhen, W.K. (2013). Antibacterial/antifungal activity and synergistic interactions between polyprenols and other lipids isolated from Ginkgo Biloba leaves. Molecules 18: 2166 - 2182.

11. Chaturvedula, V. S. P. and Prakash, I. (2012). Isolation of stigmasterol and $\beta$-sitosterol from the dichloromethane extract of Rubus suavissimus. International Current Pharmaceutical Journal, 1(9): 239 - 242.

12. Nishizawa, M., Izuhara, R., Kaneko, K. and Fujimoto, Y. (1987). 3-Caffeoyl-4-sinapoylquinic acid, a novel lipoxygenase inhibitor from Gardeniae fructus. Chemical and Pharmaceutical Bulletin, 35: 2133 - 2135.

13. Dinda, B., Debnath, S. and Majumder, S. (2005). Chemical constituents of Mussaenda incana. Indian Journal of Chemistry, 44(11): $2362-2365$.

14. Mohammed, A. M. A., Coombes, P. H., Crouch, N. R. and Mulholland D. A. (2013). Chemical constituents from Fadogia homblei de wild (Rubiaceae). International Letters of Chemistry, Physics and Astronomy, 9(2): $116-124$.

15. Lopes, M. N. L., Mazza, F. C., Maria, C. M., Young, M. C. M and Bolzani, V.S. (1999). Complete assignments of ${ }^{1} \mathrm{H}$ and ${ }^{13} \mathrm{C}$-NMR Spectra of the 3,4-seco-triterpene canaric acid isolated from Rudgea jasminoides. Journal of Brazilian Chemical Society (10)3: 237 - 240. 